\title{
Design and Performance of the ASAXS instrument at the Advanced Photon Source
}

Soenke Seifert ${ }^{1}$, Randall E. Winans ${ }^{1}$, David M. Tiede ${ }^{1}$ and P. Thiyagarajan ${ }^{2}$

'Chemistry and ${ }^{2}$ Intense Pulsed Neutron Source Divisions, Argonne National

Laboratory, 9700 South Cass Avenue, Argonne, IL 60439, USA.

Contact Author: P. Thiyagarajan, Email: thiyaga@anl.gov, FAX 630-252-416

\section{Abstract}

The SAXS instrument on the high brilliance undulator beam line (ID-12, BESSRC-CAT) at APS has been designed to produce high-resolution scattering patterns in the millisecond time domain. This instrument is equipped with a $20 \mathrm{~cm} \times 20 \mathrm{~cm}$ position sensitive gas detector and a $15 \mathrm{~cm} \times 15 \mathrm{~cm}$ high-resolution position sensitive $\mathrm{CCD}$ mosaic detector. A photodiode detector mounted on a $3 \mathrm{~mm}$ diameter beam stop permits quick alignment of the instrument as well as precise measurement of the transmitted beam intensity. The ease of changing the sample to detector distance and tuning of $\mathrm{x}$-ray energy enables easy access to different $Q$ ranges. With this instrument we routinely measure data in a $Q$ range of 0.001 to $1 \AA^{-1}$. The exposure time with the CCD detector varies from 0.1 second to $10 \mathrm{sec}$ depending on the scattering cross-section of the samples. Techniques to interface ancillary equipment for time-resolved studies and software for faster online analysis of the data have also been developed. We have obtained excellent data on the unfolding of proteins in the millisecond time domain, ASAXS of metallic alloys by using this instrument.

The submitted manuscript has been created by the University of Chicago as Operator of Argonne National Laboratory ("Argonne") under Contract No. W-31-109-ENG-38 with the U.S. Department of Energy. The U.S. Government retains for itself, and others acting on its behalf, a paid-up, nonexclusive, irrevocable worldwide license in said article to reproduce, prepare derivative works, distribute copies to the public, and perform publicly and display publicly, by or on behalf of the Government. 


\section{DISCLAIMER}

This report was prepared as an account of work sponsored by an agency of the United States Government. Neither the United States Government nor any agency thereof, nor any of their employees, make any warranty, express or implied, or assumes any legal liability or responsibility for the accuracy, completeness, or usefuiness of any information, apparatus, product, or process disclosed, or represents that its use would not infringe privately owned rights. Reference herein to any specific commercial product, process, or service by trade name, trademark, manufacturer, or otherwise does not necessarily constitute or imply its endorsement, recommendation, or favoring by the United States Government or any agency thereof. The views and opinions of authors expressed herein do not necessarily state or reflect those of the United States Government or any agency thereof. 


\section{DISCLAIMER}

Portions of this document may be illegible in electronic image products. Images are produced from the best available original document. 


\section{Introduction}

The arrival of third-generation synchrotron sources has enhanced opportunities for experiments so far not possible in condensed matter research. The high brilliance at synchrotrons such as Advanced Photon Source (APS) and ESRF will enable time-resolved studies on dynamic systems in the millisecond time domain Furthermore, it will be possible to perform experiments that require high energy resolution of the probing $\mathrm{x}$-rays and high counting statistics (e.g. anomalous SAXS). ASAXS became possible only after the advent of synchrotron sources. The power of ASAXS has been demonstrated in such areas as biophysics, tracking the disposition of trace amounts of metal atoms in proteins, to the phase separation in metallic alloys. The high brilliance of the $\mathrm{x}$-rays from the undulators at APS enables the full potential of both the time-resolved and anomalous SAXS techniques to be realized in such areas as heterogeneous catalysis, ceramics, metallic alloys, organometallics and biomaterials. We have developed a state-of-the-art SAXS instrument at APS with the capabilities for both time-resolved and anomalous SAXS applications. This paper presents the design and performance of this instrument with a few examples.

\section{Instrument Design}

The schematic of the SAXS beam line is shown in Fig.1. This instrument views a high brilliance undulator source whose characteristics (Knapp, Beno \& You, 1996) are shown in Fig. 2. This figure shows the maximum intensity of the undulator for the different sizes for the first three harmonics. We routinely use a source slit with dimension of $0.625 \mathrm{~mm} \mathrm{x}$ $0.25 \mathrm{~mm}$ in this instrument. The monochromatic beam is provided by a cryogenically 
cooled $\mathrm{Si}(111)$ double crystal monochromator. The energy can be easily tuned in the range of 5 to $28 \mathrm{KeV}$. At $45 \mathrm{~m}$ from the source we use a single mirror with three different coatings, $\mathrm{Pd}, \mathrm{Si}, \mathrm{Pt}$, to reject the $3^{\text {rd }}$ harmonic reflection from the $\mathrm{Si}(111)$ crystal as well as for vertical focussing The beam-defining slit is placed at $5 \mathrm{~m}$ down stream from the mirror. To monitor the incident $\mathrm{x}$-rays an ionization chamber placed right after the beamdefining slit is used. A shutter needed by the CCD detector is placed next to the ionization chamber. A guard slit is at $8 \mathrm{~m}$ from the beam-defining slit and this reduces the scattering from both the beam defining slit and the windows of the ionization chamber. The sample area between guard slit and the entrance to the scattering flight path is either in air or evacuated depending on the type of experiment. At the end of the scattering path is a beam stop (discussed below) mounted on a kapton film followed by the area detector

\section{Beam stop}

Fig. 3 shows the design of the beam stop that is made of lead with a hole in which a small photo diode has been mounted The beam stop has a diameter of $3.6 \mathrm{~mm}$. The electrical connections to the photo diode are made with thin $10 \mu \mathrm{m}$ wires that do not cause any significant scattering. The beam stop is mounted on a $25 \mu \mathrm{m}$ kapton film. The photodiode on the beam stop provides easy access to the perfect alignment of the beam stop. The high reliability of the photodiode provides great advantage for the on-line measurement of the transmitted beam intensity that can be readily used for the normalization of the SAXS data for the absorption of the samples and the incident flux. 


\section{Detectors}

Depending on the experiment we employ either the position sensitive 2-D gas detector designed by Andre Gabriel or a 9-segment 2-D CCD Gold detector designed by the ECT division (Westbrook and Naday, 1997) at Argonne National Laboratory. The specifications of the detector are shown in Table 1. For fast kinetic studies, where the experiment can be repeated many times, the gas detector is chosen. With this detector and a large histogramming memory of 64 Mbytes. it is possible to synchronize SAXS measurements with the sample kinetics precisely within $5 \mu \mathrm{sec}$. However, due to its count-rate limitations the beam intensity has to be attenuated by about 1000 times.

\begin{tabular}{|l|l|}
\hline Multiwire gas detector & Mosaic CCD - gold detector \\
\hline Size: $20 \mathrm{~cm} \times 20 \mathrm{~cm}$ & Size: $18 \mathrm{~cm} \times 18 \mathrm{~cm}$ \\
Pixels: $512 \times 512$ & Pixels: $3076 \times 3076$ \\
Spatial resolution $0.8 \mathrm{~mm} \times 0.8 \mathrm{~mm}$ & Pixel size: $49 \mu \mathrm{m}$ \\
Pixel resolution $0.37 \mathrm{~mm}$ & Image readout: $1.7 \mathrm{sec}$ at full size \\
Gas: $\mathrm{Ar}, \mathrm{C}_{2} \mathrm{H}_{6}$, freon & $0.5 \mathrm{sec}$ at bin size \\
Window: carbon fiber & Readout noise: 2.0 photons/pixel \\
Wires: tungsten, gold-coated & \\
Single image: $<=5 \mu \mathrm{m}$ & \\
& \\
\hline
\end{tabular}

The state-of-the-art CCD detector with 9 separate CCD's can be used with the full flux of the beam. It has a large sensitive area and the high pixel resolution that enable measurements over a wide $\mathrm{Q}$ range of about 2 orders of magnitude.

\section{Examples}

A number of interesting experiments have already been conducted by using this instrument.. A few examples are presented below to illustrate its performance. 
Fig.4 shows the SAXS data for $209 \mathrm{~nm}$ polystyrene spheres measured using the wire detector in 1 minute. The normalized SAXS data for the sample and water in $1 \mathrm{~mm}$ quartz capillary tubes are shown. The bottom curve corresponds to the background subtracted data that has a number of secondary maxima. This implies that the polystyrene spheres are monodisperse in size. We would like to point out that the photodiode within the beam stop acting as a transmission monitor enabled the proper normalization of the data even with the presence of a high background caused by the air scattering and the solution.

Time-resolved SAXS studies have been carried out by Sosnick and coworkers, University of Chicago on the unfolding of the cytochrome $\mathrm{c}$ in guadinium hydrochloride at $\mathrm{pH}$ 7. These studies were done using the gas detector and a flow cell for mixing the protein and guadinium hydrochloride solutions. These measurements were repeated for 20 cycles. In each cycle the reaction in the flow cell is initiated by a trigger pulse from the detector electronics and several measurements at intervals of 100 milliseconds were made after mixing. During the repetition of the above experiment the data from different times after mixing were stored in the 64 frames of the histogramming memory. The data were transferred to the computer after the completion of the experiment and analyzed with the software developed on the IGOR pro package on a PC.

Fig. 5 shows the radius of gyration data as a function of time after mixing of cyt $\mathrm{c}$ and 3.3 $\mathrm{M}$ guadinium hydrochloride solutions. The radius of gyration for the fully folded cytochrome $\mathrm{c}$ is around $13 \AA$. It can be seen from the figure that the radius of gyration increases rapidly within the first second and saturates at a value of $28 \AA$. These experiments were done as a feasibility study using a flow cell. Time-resolved SAXS 
studies with better time resolution are being planned by this group by using the recently acquired stop-flow apparatus with a very small dead time.

The SAXS intensity as a function of the $\mathrm{Q}$ is due to the distance correlations of all the atoms in the particles of interest. Anomalous small angle X-ray scattering (ASAXS) refers to extensions of standard SAXS experiments in which the energy of the probing Xrays are tuned near the absorption edge of an element in the sample. By performing SAXS experiments near the characteristic absorption edge of any given atom, it is possible to vary the contrast for scattering of that particular element. Dale Alexander and coworkers (Alexander, Kestel, Jemian, Odette, Klingensmith \& Gragg,), Materials Science Division at Argonne National Laboratory have recently studied the precipitation of $\mathrm{Cu}$ in Fe-0.9 Cu-1.0 Mn alloy annealed at $450^{\circ} \mathrm{C}$ for 24 hours. Fig. 6 depicts the Guinier plots of the small angle $\mathrm{x}$-ray scattering data measured at 4 energies just below the k-edge of $\mathrm{Fe}(7112 \mathrm{eV})$. It is clearly seen that as the incident photon energy increases near the absorption edge, the scattering contrast between $\mathrm{Cu}$-rich precipitates and the $\mathrm{Fe}$ matrix increases resulting in a monotonic increase in the SAXS intensity with increasing energy.

\section{$\underline{\text { Summary }}$}

The state-of-the art SAXS instrument developed at the BESSRC-CAT at APS is versatile to carry out time -resolved and anomalous SAXS experiments in condensed matter research. This instrument provides easy access to a wide $Q$ region through both tuning of the probing $\mathrm{x}$-rays and the changing of the sample-to-detector distance. The photo diode 
on the beam stop enables accurate measurement of the transmitted beam and the scattering data from the area detector can be properly normalized for the incident beam flux and the absorption. In essence both scattering and the transmission data are obtained in a single measurement. The background of the instrument has been reduced by spacing the slits at large distances and evacuation of the beam line. This instrument has a number of ancillary equipment to vary the sample environment. The performance of this instrument has been illustrated with a few examples.

\section{Acknowledgement}

This work was performed under the auspices of the Office of Basic Energy Sciences; Division of Chemical and Division of Materials Sciences, U.S. Department of Energy, under contract number W-31-109-ENG-38. The support of the BESSRC staff is appreciated, especially Dr. Jennifer Linton, Dr. Mark Beno, Dr. Guy Jennings and Mark Engbretson. We are indebted to Dr. Gregory Beaucage, University of Cincinnati for providing the data reduction programș on Igor Pro and Dr. Ken Littrell, IPNS for developing additional analysis software, Dr. Dale Alexander, MSD and Professor Tobin Sosnick, Department of Biochemistry and Molecular Biology for remitting us to use their data as examples.

\section{Figure Legends}

Fig.1. Tuning curves for the flux through a series of apertures of different size at $30 \mathrm{~m}$ from the source. Pinhole sizes are: $5.0 \mathrm{~mm} \times 2.0 \mathrm{~mm}$ (solid), $2.5 \mathrm{~mm} \times 1.0 \mathrm{~mm}$ (dotted), $1.25 \mathrm{~mm} \times 0.5 \mathrm{~mm}$ (dashed), and $0.625 \mathrm{~mm} \times 0.25 \mathrm{~mm}$ (dash-dotted)

Fig.2. Beam line and small angle set-up. The values behind the description are the 
distances from the source.: 1.) Detector, $68 \mathrm{~m}$; 2.) Beam stop, $67 \mathrm{~m}$; 3.) Scattering beam path, from $58 \mathrm{~m}$ to $67 \mathrm{~m}$; 4.) Sample, $58 \mathrm{~m}$; 5.) Guard slit (pinhole), $58 \mathrm{~m}$; 6.) Shutter, $57 \mathrm{~m}$; 7.) Ionization chamber, $52 \mathrm{~m}$; 8.) Beam defining slit, $50 \mathrm{~m}$; 9.) Horizontal mirror, $45 \mathrm{~m}$; 10.) Pinhole, source defining slit, $38 \mathrm{~m}$; 11.) Double monochromator, $35 \mathrm{~m}$; 12.) Vertical mirror, $33 \mathrm{~m}$; 13.) Undulator

Fig.3. Beam stop with built in photo diode for transmission monitoring.

Fig.4. $\log -\log$ plot of the SAXS data from $209 \mathrm{~nm}$ polystyrene sphere solution and water in $1 \mathrm{~mm}$ capillary tubes and the background subtracted data. The appearance of a number of secondary maxima in the data indicate that the spherical particles are monodisperse in size.

Fig.5. Time-resolved unfolding of cytochrome $\mathrm{c}$ in $3.3 \mathrm{M}$ guadinium hydrochloride solution.

Fig.6. ASAXS data measured near the $\mathrm{K}$ edge of $\mathrm{Fe}$ for the $\mathrm{Fe}-0.9 \mathrm{Cu}-1.0 \mathrm{Mn}$ alloy annealed at $450^{\circ} \mathrm{C}$ for 24 hours,

\section{References}

Alexander, D. E., B.J. Kestel, B.J., Jemian, P.R.,. Odette, G.R., Klingensmith, D. \& Gragg, D. in Microstructural Processes in Irradiated Materials, Vol. 540, edited by S.J. Zinkle, S.J., Lucas, G.E., Ewing , R.C. \& Williams, J.S. (Materials Research Society, Warrendale, PA, 1999), pp. 415-463.

Knapp, G.S., Beno, M.A. \& You, H, (1996) Annu. Rev. Mater Sci, 26, 693-725.

Westbrook, E. \& Naday, S. (1997) Methods Enzymol. 276, 244. 


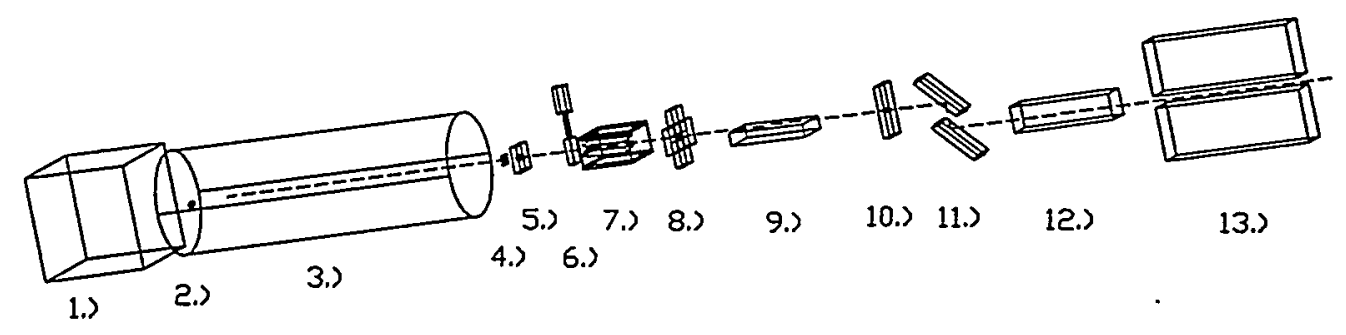

Fig.1

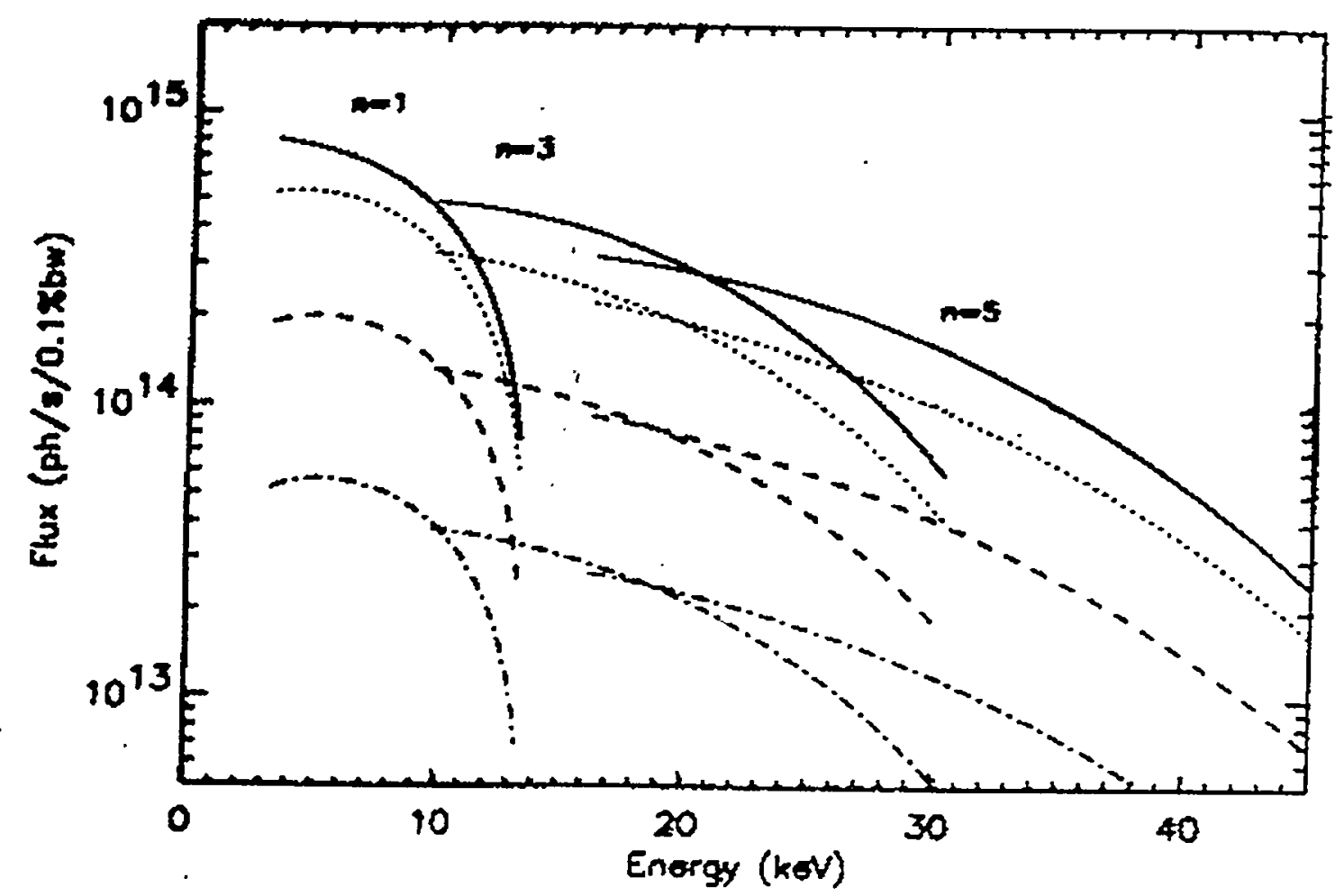

Fig.2. 


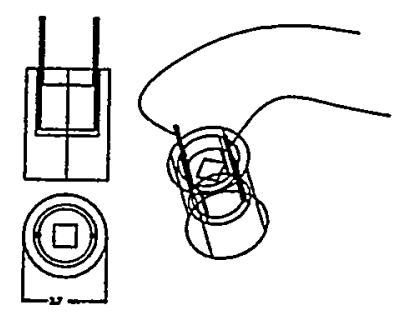

Fig3

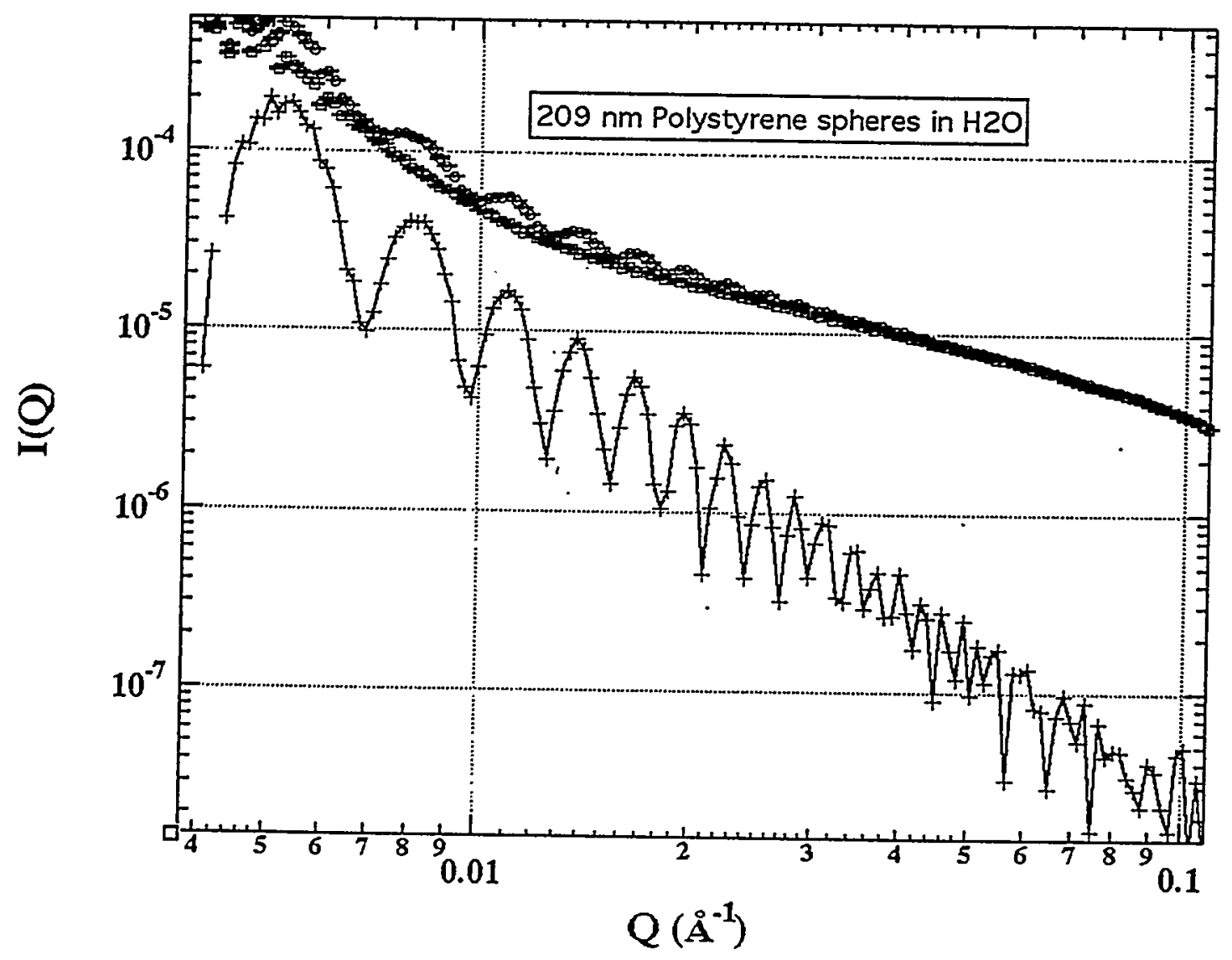

Fig4 


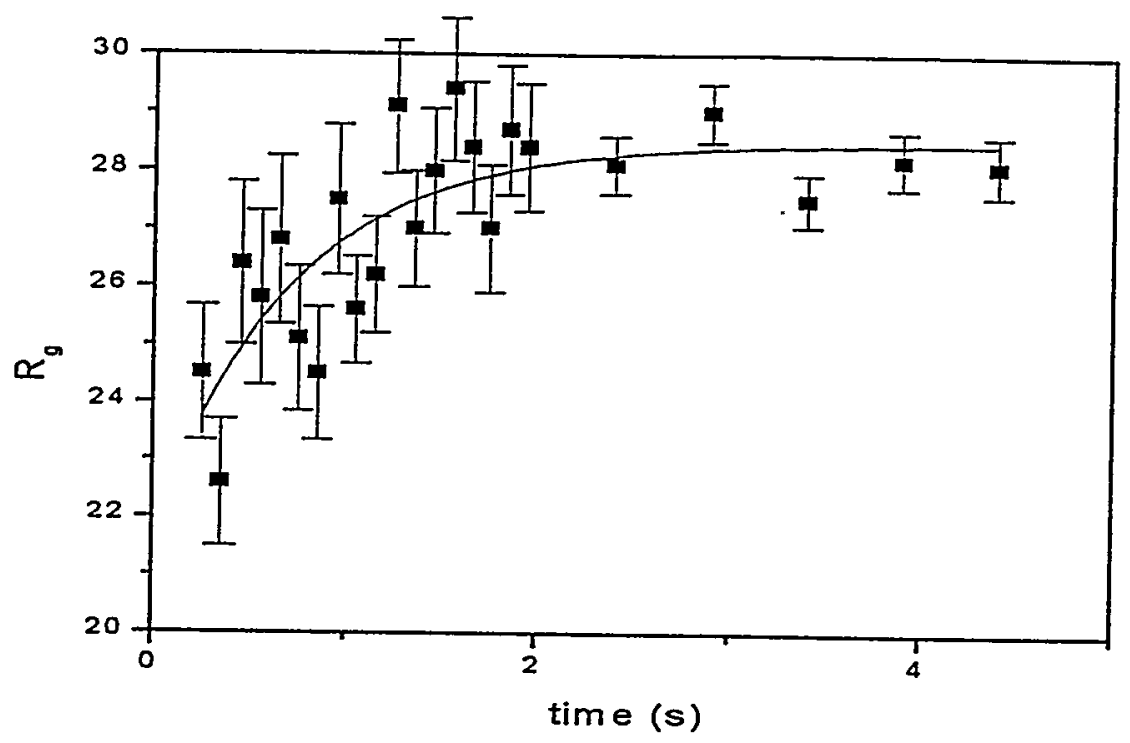

Fig5

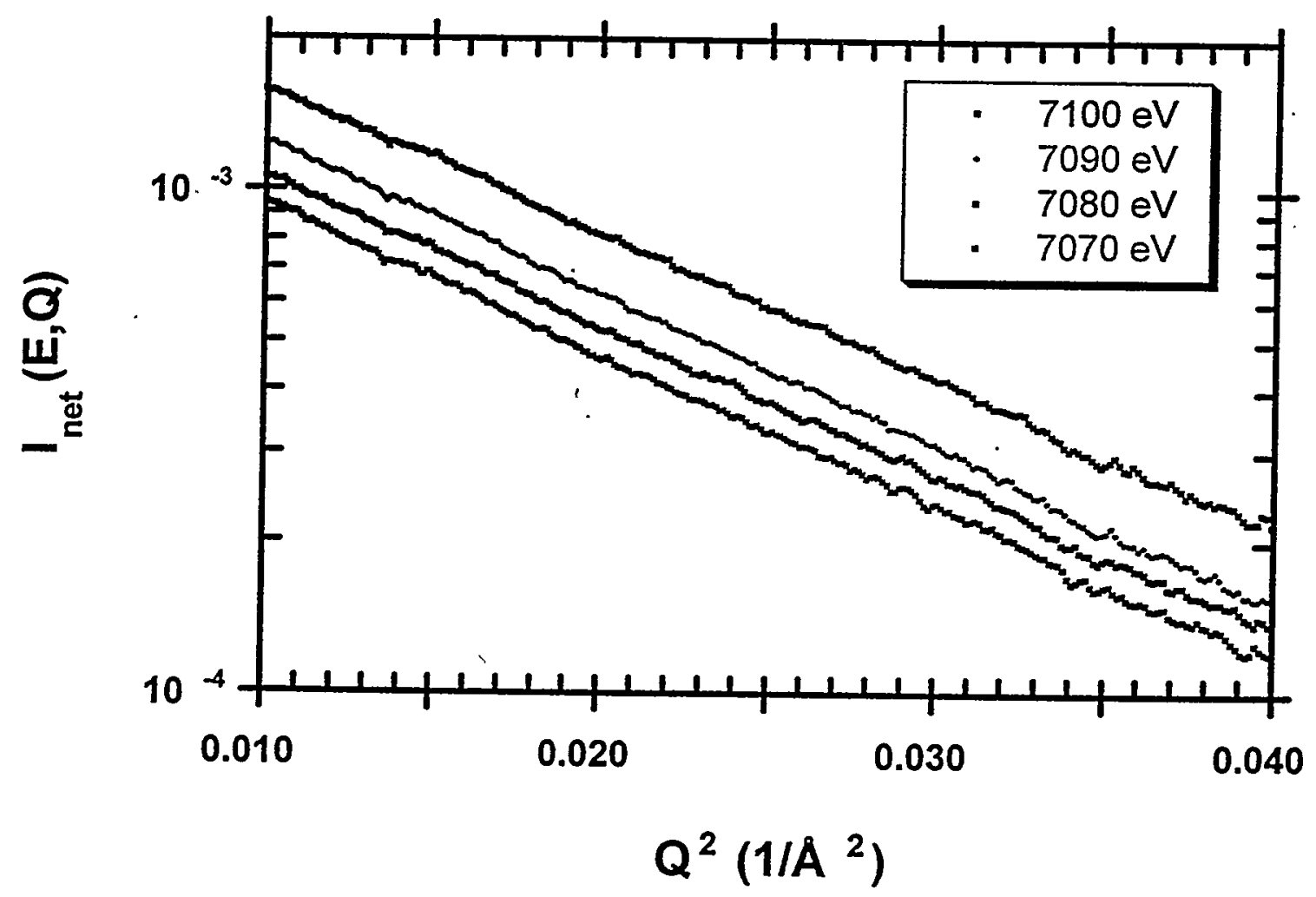

Fig.6 